Photodetachment microscopy to an excited spectral term and the electron affinity of phosphorus

This article has been downloaded from IOPscience. Please scroll down to see the full text article.

2011 J. Phys. B: At. Mol. Opt. Phys. 44195009

(http://iopscience.iop.org/0953-4075/44/19/195009)

View the table of contents for this issue, or go to the journal homepage for more

Download details:

IP Address: 161.111.22.141

The article was downloaded on 11/12/2012 at 11:14

Please note that terms and conditions apply. 


\title{
Photodetachment microscopy to an excited spectral term and the electron affinity of phosphorus
}

\author{
R J Peláez ${ }^{1}$, C Blondel, M Vandevraye, C Drag and C Delsart \\ Laboratoire Aimé-Cotton, Centre national de la recherche scientifique, université Paris-sud, \\ bâtiment 505, F-91405 Orsay cedex, France \\ E-mail: christophe.blondel@u-psud.fr
}

Received 27 June 2011, in final form 25 August 2011

Published 21 September 2011

Online at stacks.iop.org/JPhysB/44/195009

\begin{abstract}
A beam of $\mathrm{P}^{-}$ions produced by a cesium sputtering ion source was photodetached in the presence of an electric field, with a single-mode ring dye laser. Neutral $\mathrm{P}$ can be produced at one or the other of the fine-structure sub-levels of its $3 \mathrm{~s}^{2} 3 \mathrm{p}^{3}{ }^{2} \mathrm{D}^{\mathrm{o}}$ excited term. This is the first atomic photodetachment microscopy experiment with excitation of the parent neutral atom out of the fundamental spectral term. The background electron signal due to ground-state photodetachment notwithstanding, photodetachment microscopy images produced at the excited thresholds could be analysed to provide a measure of these excited-term thresholds with interferometric precision. Starting from the three possible fine-structure sub-levels of $\mathrm{P}^{-} 3 \mathrm{~s}^{2} 3 \mathrm{p}^{4}{ }^{3} \mathrm{P}$, the five fine-structure thresholds that may be detected, taking the selection rules into account, have been measured. They are combined with the spectroscopic data available in the literature on neutral $\mathrm{P}$ to produce an improved experimental value of the electron affinity ${ }^{e} A$ of phosphorus: $602179(8) \mathrm{m}^{-1}$ or $0.746607(10) \mathrm{eV}$. Taking all covariances of the optimized energy levels into account, one can merge them with the former measure of the three lowest detachment thresholds of $\mathrm{P}^{-}$, which results in a slightly more precise value of ${ }^{e} A(\mathrm{P}): 602181(8) \mathrm{m}^{-1}$, or $0.746609(9) \mathrm{eV}$. The accuracy of ${ }^{e} A(\mathrm{P})$ is now essentially limited by the uncertainty on the ${ }^{2} \mathrm{D}_{3 / 2}^{\mathrm{o}}$ and ${ }^{2} \mathrm{D}_{5 / 2}^{\mathrm{o}}$ energy levels of the neutral atom. The fine-structure intervals of the $3 \mathrm{~s}^{2} 3 \mathrm{p}^{3}{ }^{2} \mathrm{D}^{\mathrm{o}}$ doublet of the neutral atom and of the $3 \mathrm{~s}^{2} 3 \mathrm{p}^{4}{ }^{3} \mathrm{P}$ triplet of the negative ion have their accuracy improved by more than one order of magnitude.
\end{abstract}

(Some figures in this article are in colour only in the electronic version)

\section{Introduction}

Photodetachment microscopy [BDD96], in addition to being one of the most direct visualizations of a matter wave, has now been the most precise method for electron affinity measurements for ten years. Experimental electron affinities [AHH99] are a stringent test of the theorists' ability to calculate the eigenstates of highly correlated systems. Even though $a b$ initio calculations seldom yield more than three reliable digits [dOMdPG99, PM11], measuring an atomic electron affinity with a better than $10^{-6}$ accuracy can serve as a test of isotope shift calculations, as was, e.g., done for the electron

1 Present address: Instituto de Optica, CSIC, Madrid, Spain. affinities of hydrogen [LML91], chlorine [BGH+95], oxygen $[\mathrm{BDV}+01]$ and, more recently, sulfur [CDS+10]. At this level of accuracy, photodetachment to excited states may also give valuable spectroscopic information on the spectrum of the parent neutral atom, especially when dealing with excited levels that cannot be directly electric-dipole excited from the atom ground state.

The photodetachment microscope has just been equipped with a cesium sputtering ion source. This opens the way to the production of a greater variety of atomic negative ions than with the old-fashioned low-energy hot-cathode ion source [BDDV99]. This new set-up is used to study photodetachment microscopy on a beam of $\mathrm{P}^{-}$ions. 
Phosphorus has a rather low electron affinity ${ }^{e} A(\mathrm{P})$ of about $0.75 \mathrm{eV}$, or $6 \times 10^{5} \mathrm{~m}^{-1}$. The first excited term of neutral $\mathrm{P}, 3 \mathrm{~s}^{2} 3 \mathrm{p}^{3}{ }^{2} \mathrm{D}^{\mathrm{o}}$ lying more than $10^{6} \mathrm{~m}^{-1}$ above the ground level, gives rise to higher detachment thresholds well suited for the photodetachment by a dye laser. Excitation of the neutral atom can also enhance core-electron rescattering effects, which have up to now been found to be too small to influence the low-energy photoelectron interferences studied experimentally [Fab94]. It is only in the presence of a resonance that one actually expects a strong perturbation of the photodetached electron wave. But the interferograms recorded in photodetachment microscopy give a measure of the photoelectron ejection energy with interferometric precision; checking the consistency of kinetic energy measurements performed at different excitation energies can thus reveal even relatively slight perturbations. The absence of any perturbation would leave the way open to new electron affinity measurements, via excited states. This is the kind of experiment we report about in phosphorus.

\section{Experimental set-up}

\subsection{Ion beam and detection}

The photodetachment microscope [BDDV99], initially equipped with a hot-cathode discharge source and a oneby-one electron detector based on a resistive anode, is now equipped with the SNICS II cesium sputtering ion source ${ }^{2}$ and an optical-imaging detector. The essential improvement brought in by the latter is the capacity to record electron interferograms even with pulsed-laser excitation, as was demonstrated in the recent measurement of the electron affinity of iodine [PBDD09]. This work is the first application of the SNICS ion source for photodetachment microscopy.

One objective of the ion beam preparation being to make the Doppler effect as small as possible in the interaction region, the extraction voltages of the sputtering ion source are kept as low as possible in the regime where a stable ion beam can be produced. This still implies having typically a $-12 \mathrm{kV}$ voltage between the source body and the extractor and another $-5 \mathrm{kV}$ between the extractor and the cathode (where $\mathrm{Cs}^{+}$ sputtering takes place). As a consequence, the ion beam so produced has to be decelerated by $15.8 \mathrm{kV}$ to have its kinetic energy reduced to the usual $1.2 \mathrm{keV}$ compatible with our ion optical elements. This first deceleration stage takes place at the exit of the source chamber. Numerical simulations have shown that even after we have reset the internal voltages of the decelerator so as to minimize the divergence effect linked with deceleration, the deceleration stage still significantly reduces the ion current that can pass inside the $1 \mathrm{~mm}$ diameter left open by the subsequent diaphragms. The $\mathrm{P}^{-}$ion current finally available is thus reduced to about $100 \mathrm{pA}$, after the final deceleration down to $300 \mathrm{eV}$, in the interaction region.

2 National Electrostatics Corp. 7540 Graber Road, PO Box 620310, Middleton, WI 53562-0310 USA.

\subsection{Laser}

Photodetachment of $\mathrm{P}^{-}$is achieved by means of a $\mathrm{CW}$ ring dye laser (Spectra-Physics 380) pumped by the $514 \mathrm{~nm}$ line of an argon-ion laser. The wavenumbers of interest range from 1711800 to $1739900 \mathrm{~m}^{-1}$ due to the exploration of the fine structure (see below), quite suitable for the use of Rhodamine 590. The laser wavenumber is servo-locked on a home-made sigmameter [JP75] to a small fraction of the latter's $0.5 \mathrm{~m}^{-1}$ free spectral range. This wavenumber is continuously measured with an accuracy better than $0.1 \mathrm{~m}^{-1}$ by a HighFinesse WS-8 lambdameter. Calibration of the lambdameter is checked several times every day with the light of a diode set on a well-known saturated absorption component of the D2 line of atomic Cs.

In order to minimize the Doppler shift, the laser beam should illuminate the ion beam at right angles with respect to the ion velocity. The presence of the electron detector in this very direction has obliged us to shift the laser beam axis by a few degrees, which results in a not quite negligible Doppler shift. Elimination of this Doppler shift is made possible by passing the laser twice on the ion beam, back and forth, and calculating an average of the measured energies [BDG01].

\section{Spectroscopic data on the $\mathbf{P}^{-}$-P system}

\subsection{The electron affinity of phosphorus}

The electron affinity ${ }^{e} A(\mathrm{P})$ was measured several times, together with the other detachment thresholds from the ${ }^{3} \mathrm{P}$ sublevels, by the 'laser photodetachment threshold' (LPT) technique, which consists in monitoring the onset of photodetachment while scanning the laser frequency [Fel76, SL77, ALA+07]. Several detachment thresholds can be measured by recording the onset of new contributions to the photodetachment signal as the excitation wavenumber is increased. When the photodetachment experiment is carried out in the infrared, the threshold from ${ }^{3} \mathrm{P}_{2}$, labelled $S$ on figure 1, directly gives ${ }^{e} A(\mathrm{P})$. In the most recent LPT experiment [ALA+07], the obtained value was $602240(50) \mathrm{m}^{-1}$ or $0.74668(6) \mathrm{eV}$.

The $a b$ initio calculation of ${ }^{e} A$ was investigated in several recent articles, the most successful of which, as far as the proximity of the result to the experimental value may serve a criterion, are those by Guo-xin et al [GxOT98] and de Oliveira et al [dOMdPG99]. These calculated values are compared to the experimental ones in table 1.

\subsection{The P I spectrum}

Our knowledge of the P I spectrum relies on the spectrum and term analysis performed by Svendenius in 1979 [Sve80]. The levels of phosphorus reached in our photodetachment experiment are the fine-structure sublevels of the excited D term of the ground configuration $1 \mathrm{~s}^{2} 2 \mathrm{~s}^{2} 2 \mathrm{p}^{6} 3 \mathrm{~s}^{2} 3 \mathrm{p}^{3},{ }^{2} \mathrm{D}_{3 / 2}^{\mathrm{o}}$ and ${ }^{2} \mathrm{D}_{5 / 2}^{\mathrm{o}}$, with excitation energies of 1136102 and $1137663 \mathrm{~m}^{-1}$, respectively. Energies given down to the $1 \mathrm{~m}^{-1}$ digit are told to have an 'estimated error' of 1 to $5 \mathrm{~m}^{-1}$. We shall assume, as the most careful hypothesis, that these energies are known 


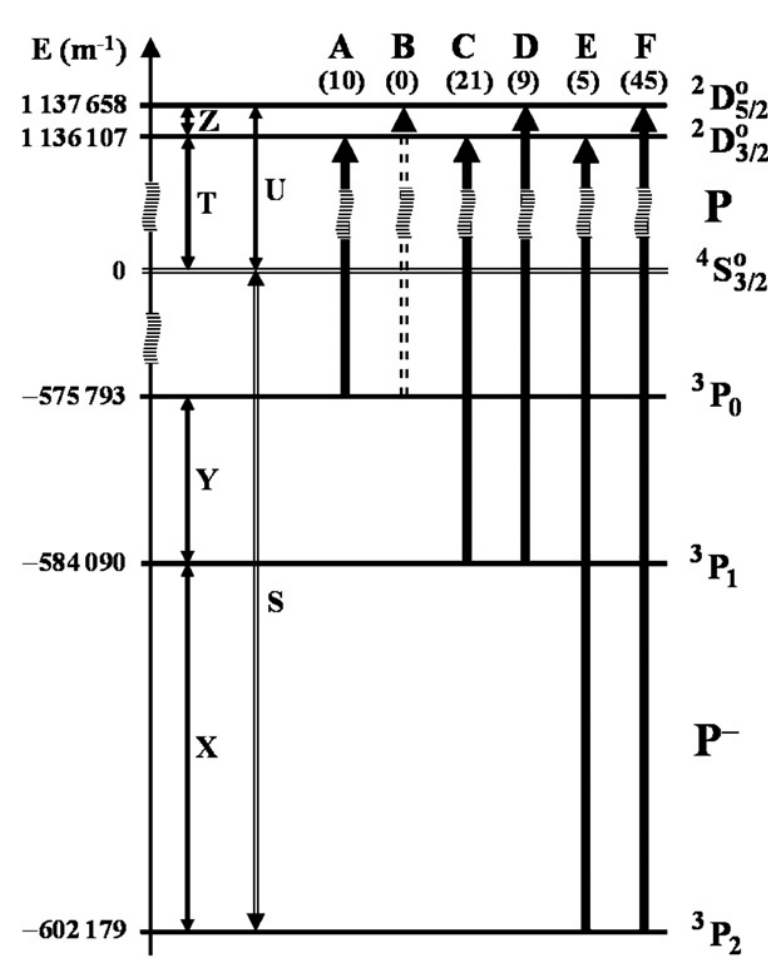

Figure 1. The fine structure of $\mathrm{P}^{-}$and the ${ }^{2} \mathrm{D}$ excited level of $\mathrm{P}$, with the ${ }^{4} \mathrm{~S}^{\mathrm{o}}$ level of the neutral in between. $\mathrm{A}^{3} \mathrm{P} \rightarrow{ }^{2} \mathrm{D}^{\mathrm{o}}$ doublet photodetachment scheme gives rise to a fine structure of six thresholds (if one includes the electric-dipole forbidden ${ }^{3} \mathrm{P}_{0} \rightarrow{ }^{2} \mathrm{D}_{5 / 2}^{\mathrm{o}}$ one), which we label $A$ to $F$ in the order of increasing energies. The relative intensities, calculated assuming pure $L S$ coupling [BCDD06], are given by an integer number below each label. The figure also gives the definition of the independent spectroscopic quantities: the fine-structure intervals $X, Y$ and $Z$, the electron affinity ${ }^{e} A(\mathrm{P}) \equiv S$, and the first energy levels of neutral phosphorus $T$ and $U$.

Table 1. Calculated and measured values (the latter with uncertainty) of the electron affinity of phosphorus ${ }^{e} A(\mathrm{P})$. All previous measurements used the laser-photodetachment-threshold (LPT) technique; the first and the last [Fel76, ALA+07] used a laser pumped optical parametric oscillator to directly select the ground ${ }^{4} \mathrm{~S}_{3 / 2}^{o}$ level of P, while the intermediate one [SL77] applied the LPT technique to the same excited ${ }^{2} \mathrm{D}^{\mathrm{o}}$ thresholds as in this study.

\begin{tabular}{ll}
\hline${ }^{e} A(\mathrm{eV})$ & Reference \\
\hline 0.702 & [WP98] \\
0.714 & [GJB98] \\
0.748 & [GxOT98] \\
0.7467 & [dOMdPG99] \\
$0.74676_{-50}^{+30}$ & [Fel76] \\
$0.7464(4)$ & [SL77] \\
$0.74668(6)$ & [ALA+07] \\
$0.746607(10)$ & present work (2011) \\
\hline
\end{tabular}

with a $5 \mathrm{~m}^{-1}$ standard deviation. As far as the $3 \mathrm{~s}^{2} 3 \mathrm{p}^{3}{ }^{2} \mathrm{D}^{\mathrm{o}}$ doublet is concerned, this will prove to be quite consistent with the results.

\subsection{Hyperfine structure}

${ }^{31} \mathrm{P}$ is the only stable isotope of phosphorus, with a nuclear spin $I=1 / 2$, which gives rise to hyperfine doublets for all fine-structure levels but the $J=0$ ones. The hyperfine structure (hfs) is usually not resolved by photodetachment microscopy, which thus produces an incoherent sum of the interferograms corresponding to every possible hyperfine subthreshold. In order to fit the experimental interferograms with synthetic ones, the best method is to use the hyperfine splittings taken from the literature, to make a rigorous reconstruction of the expected electron image. This way the data analysis was carried out for the photodetachment microscopy of negative ions with a hyperfine structure, most recently of ${ }^{127} \mathrm{I}^{-}$ [PBDD09]. Unfortunately nothing is known of the hfs of the ${ }^{2} \mathrm{D}^{\mathrm{o}}$ term of the $3 \mathrm{~s}^{2} 3 \mathrm{p}^{3}$ configuration of phosphorus, nor of the hfs of the ${ }^{31} \mathrm{P}^{-}$anion.

Extrapolating the hfs of ${ }^{31} \mathrm{P}^{-}$from the hfs of the isoelectronic ${ }^{33} \mathrm{~S}$ atom, in a way already used with some success to estimate the hfs of ${ }^{33} \mathrm{~S}^{-}$from the hfs of $\mathrm{Cl}$ [TJL89] or the hfs of ${ }^{17} \mathrm{O}^{-}$from the hfs of ${ }^{19} \mathrm{~F}[\mathrm{BDV}+01]$, has just become possible thanks to a very recent $a b$ initio calculation of the hfs of ${ }^{33} \mathrm{~S}$ [CG11]. The method consists in rescaling the hyperfine splitting of the isoelectronic neutral, considering that most of it is due to the magnetic dipole term, through a multiplication by the nuclear Landé factor and fine-structure splitting ratios. This provides an estimated magnetic dipole hyperfine constant of about $313 \mathrm{MHz}$ for the ${ }^{3} \mathrm{P}_{2}$ level and only $-11 \mathrm{MHz}$ for the ${ }^{3} \mathrm{P}_{1}$ level of ${ }^{31} \mathrm{P}^{-}$. The ${ }^{3} \mathrm{P}_{0}$ level has of course no hyperfine structure.

The hyperfine structure of atomic phosphorus is known only for the fundamental term ${ }^{4} S_{3 / 2}^{o}$ [LP62, LP63, PS64, DMPD68] and for some even-parity excited levels, where it is particularly large for configurations with an unpaired s electron [Sve80, SV80]. Our analysis will thus be carried out as in the absence of any hfs, only allowing for a slightly increased spectral broadening, with the expectation of a significantly broader figure for all photodetachment experiments starting from the ground ${ }^{3} \mathrm{P}_{2}$ level of $\mathrm{P}^{-}$. The measured detachment thresholds shall be understood as hyperfine-averaged ones.

\section{Data analysis}

\subsection{Detachment threshold measurements}

An example of a photoelectron interferogram is given by figure 2. In order to consolidate the photodetachment microscopy measurement of a detachment energy, the threshold value is not deduced from a single measurement of the photoelectron energy $\epsilon$. Series of photodetachment microscopy interferograms are recorded at all energies compatible with the spatial resolution of the interference rings (on the higher energy side) and the observation of at least one ring (on the lower energy side) [BDDV99]. The detachment threshold is determined as the extrapolation of the measured threshold at $\epsilon=0$. An error made when estimating the experimental electric field will make the apparent threshold vary as a function of $\epsilon$, with a derivative just equal to $-2 / 3$ of the relative error on the electric field. Experimentally this derivative, or slope of the regression line, always lies within the $\pm 3 \%$ interval [BCD+05].

The example of the data set recorded for threshold $\mathrm{F}$ is given by figure 3 . Similar linear regressions are made for 

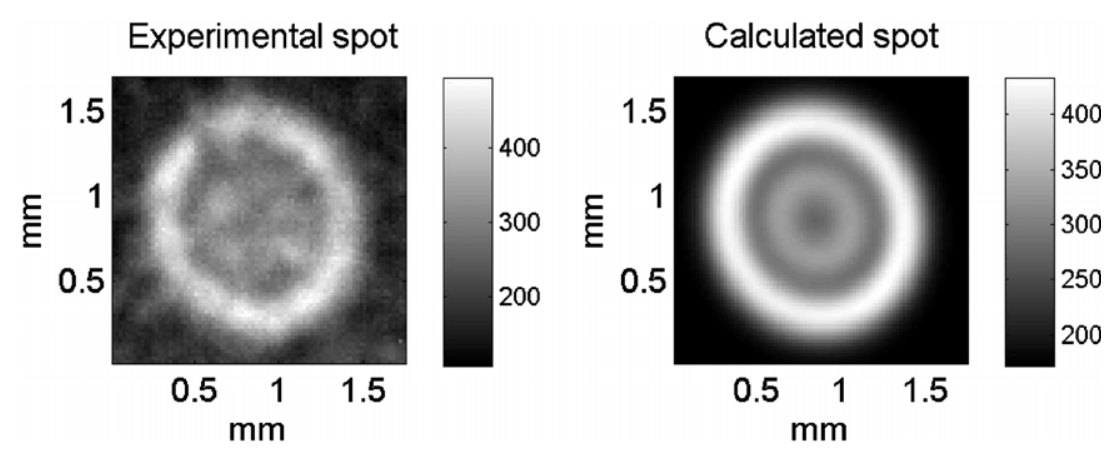

Figure 2. Example of an experimental photodetachment spot in an electric field of $364.5 \mathrm{Vm}^{-1}$ (a) and the best-fitting theoretical electron interferogram (b), calculated for an initial electron kinetic energy of 53.1(2) $\mathrm{m}^{-1}$. Subtracting this energy from the laser wavenumber of $1721796.4 \mathrm{~m}^{-1}$ would directly yield the detachment threshold (here threshold D) were it not for the Doppler shifting of the laser frequency in the ions frame. Comparison of the raw difference of 1721743.4 with the result of the final adjustment of threshold $\mathrm{D}$ at $1721748.0 \mathrm{~m}^{-1}$ reveals that in this very case, the photon energy, as seen by the ions, was positive-shifted by $4.6 \mathrm{~m}^{-1}$. A $1.1^{\circ}$ deviation from orthogonality between the laser and ion beam is enough to produce a shift of this magnitude. Simultaneous photodetachment to the lower fine-structure ${ }^{2} \mathrm{D}_{3 / 2}^{\mathrm{o}}$ level and to the ground ${ }^{4} \mathrm{~S}_{3 / 2}^{\mathrm{o}}$ level of phosphorus always produce a photoelectron background, but the higher energy of these photoelectrons causes their interference pattern to be completely smoothed out. Moreover, the corresponding signal is spread on a surface proportional to the kinetic energy, which even makes it unnecessary to subtract the corresponding background (here only 172 in arbitrary units).

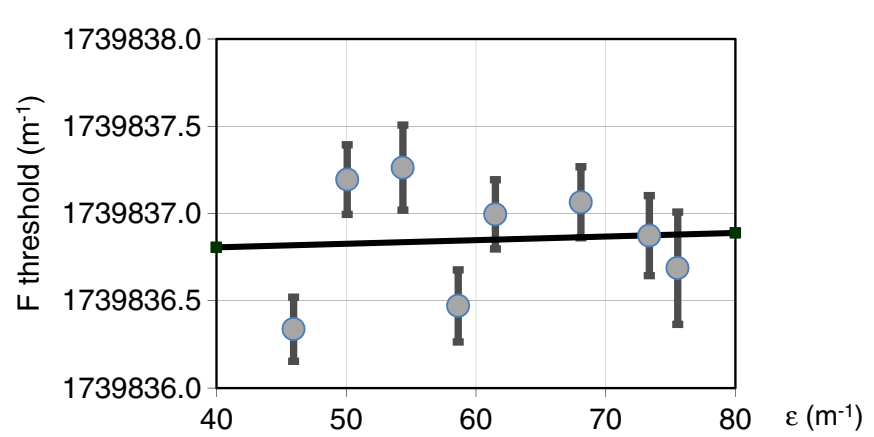

Figure 3. The apparent detachment energy at threshold $\mathrm{F}$, for mean photoelectron kinetic energies $\epsilon$ ranging from 46 to $76 \mathrm{~m}^{-1}$. Extrapolation down to $\epsilon=0$ produces a measurement of the photodetachment threshold, either $1739836.6(9) \mathrm{m}^{-1}$ taking only this set of data into account or $1739836.7(6) \mathrm{m}^{-1}$ taking the necessary similarity between all regression slopes into account (see the text). The experimental error on the electric field, as deduced from this slope, is $-0.14(26) \%$.

all of the studied detachment thresholds. A single leastsquares fitting procedure can be applied to all five sets of the data together, with the slope differences constrained by an arbitrary slope-difference standard deviation. Constraining the different slopes to be similar is justified by the assumption that the relative error on the electric field remains about the same for the whole series of experiments. Observations made in previous studies have suggested that a $0.5 \%$ standard deviation of the slope is a conservative estimate of the residual variations, which may be due to the day-to-day variations of the ion beam positioning in the interaction chamber.

Coupling the five detachment threshold measurements in this way has the consequence of (i) increasing the precision of each threshold and (ii) introducing a correlation between the experimental threshold variances. The corresponding correlation coefficients (covariances divided by the geometrical average of the corresponding variances) are of course positive, because compelling the slopes to remain
Table 2. Measured thresholds with their $2 \sigma$ statistical uncertainties. The readjusted values take the internal consistency of the level scheme into account (which has only four independent energy levels). The readjustment also incorporates the additional constraint on the ${ }^{2} \mathrm{D}^{\circ}$ fine structure given by the spectroscopy of neutral $\mathrm{P}$ [Sve80], which explains the reduction of the uncertainty for all cases but threshold A. Threshold A, due the forbidden character of the B threshold, has no direct counterpart to the upper ${ }^{2} \mathrm{D}_{5 / 2}^{\mathrm{o}}$ sublevel, hence, no direct coupling to the ${ }^{2} \mathrm{D}^{\circ}$ fine-structure measurement.

\begin{tabular}{lll}
\hline Transition & Measured threshold $\left(\mathrm{m}^{-1}\right)$ & Readjusted value $\left(\mathrm{m}^{-1}\right)$ \\
\hline$A$ & $1711899.94(54)$ & $1711899.94(54)$ \\
$C$ & $1720197.49(56)$ & $1720197.47(55)$ \\
$D$ & $1721747.97(53)$ & $1721747.99(49)$ \\
$E$ & $1738286.16(78)$ & $1738286.20(63)$ \\
$F$ & $1739836.72(49)$ & $1739836.72(48)$ \\
\hline
\end{tabular}

similar produces a trend, for all intercepts, to vary in a positively correlated way. The correlation coefficients of all pairs of measured thresholds actually remain in the 48-61\% range. The variance-covariance matrix $\Sigma_{T}$ of the measured thresholds shall be taken into account for the subsequent transformation of the threshold values into a measurement of ${ }^{e} A$ on the one hand, and the internal fine-structure energies, both of the neutral and the anion, on the other hand. The results of all five threshold measurements are given in table 2. Unless otherwise stated, the uncertainties quoted throughout the paper correspond to two standard deviations.

\subsection{Data analysis and covariance algebra}

The thresholds just measured are of course linear functions of the fundamental intervals defined by figure 1 . Because our threshold measurements will question the neutral phosphorus spectrum via the new estimate they give of the ${ }^{2} \mathrm{D}^{\circ}$ fine structure, it appears useful to extend this point of view to include Svendenius's measurements [Sve80] and say that the seven-component vector $M$ composed of the five thresholds 
Table 3. Optimized energy intervals in the $\mathrm{P}^{-}$or $\mathrm{P}$ system. The first five lines are the independent components of vector $\boldsymbol{X}$, as they come out of the calculation.

\begin{tabular}{llll}
\hline Level & Energy $\left(\mathrm{m}^{-1}\right)$ & $\begin{array}{l}\text { Standard } \\
\text { deviation } \sigma\end{array}$ & $\begin{array}{l}\text { Former } \\
\text { value }\end{array}$ \\
\hline$X \equiv{ }^{3} \mathrm{P}_{1}-{ }^{3} \mathrm{P}_{2}$ & 18088.73 & 0.19 & 18135 \\
$Y \equiv{ }^{3} \mathrm{P}_{0}-{ }^{3} \mathrm{P}_{1}$ & 8297.52 & 0.25 & 8265 \\
$Z \equiv{ }^{2} \mathrm{D}_{5 / 2}^{\mathrm{o}}-{ }^{2} \mathrm{D}_{3 / 2}^{\mathrm{o}}$ & 1550.52 & 0.20 & 1561 \\
$S \equiv{ }^{2} A$ & 602178.96 & 3.55 & 602235 \\
$T \equiv{ }^{2} \mathrm{D}_{3 / 2}^{\mathrm{o}}$ & 1136107.24 & 3.54 & 1136102 \\
$X+Y \equiv{ }^{3} \mathrm{P}_{0}-{ }^{3} \mathrm{P}_{2}$ & 26386.25 & 0.28 & 26400 \\
$T+Z \equiv{ }^{2} \mathrm{D}_{5 / 2}$ & 1137657.76 & 3.54 & 1137663 \\
\hline
\end{tabular}

under scrutiny and the two relevant excitation energies of neutral $\mathrm{P}$ is a linear function of the five-component vector $\boldsymbol{X}={ }^{\mathrm{t}}(X, Y, Z, S, T)$. In matrix form $\boldsymbol{M}=\boldsymbol{I} \boldsymbol{X}$, where $\boldsymbol{I}$ is a $7 \times 5$ matrix (with zeros, 1 and -1 coefficients that describe the energy sums and differences). The question is to determine the most probable value of $\boldsymbol{X}$, the reconstructed value of $\boldsymbol{M}$, and the related uncertainties.

The $5 \times 5$ covariance matrix $\boldsymbol{\Sigma}_{T}$ associated with our threshold measurements can be extended to a $7 \times 7$ covariance matrix $\boldsymbol{\Sigma}$ associated with the measured vector $\boldsymbol{M}_{\exp }$. It has of course zero covariances between our measurements and the spectroscopic data on neutral phosphorus. The leastsquares optimization of the energy levels, in an algebraic form that has been well known for 40 years [Kra11, RFSG72], provides the best-fitting $\boldsymbol{X}$ vector as the one that minimizes the quadratic form ${ }^{t}\left(\boldsymbol{I} \boldsymbol{X}-\boldsymbol{M}_{\exp }\right) \boldsymbol{N}\left(\boldsymbol{I} \boldsymbol{X}-\boldsymbol{M}_{\text {exp }}\right)$, where $\boldsymbol{N}=$ $\Sigma^{-1}$. The nullity condition of its gradient straightforwardly provides the coordinates of the minimum, explicitly $\boldsymbol{X}=$ $\left.{ }^{t} \boldsymbol{I N I}\right)^{-1 t} \boldsymbol{I N} \boldsymbol{M}_{\text {exp. }}$. The numerical result for this $\boldsymbol{X}$ vector is given in table 3 , together with other energy intervals of interest that can be deduced from the $\boldsymbol{X}$ components. The $\boldsymbol{X}$ covariance matrix, in $\mathrm{m}^{-2}$, is given by

$$
\begin{aligned}
\left({ }^{t} \boldsymbol{I N I}\right)^{-1} & \\
= & \left(\begin{array}{ccccc}
0.037 & -0.010 & -0.013 & 0.024 & 0.006 \\
-0.010 & 0.061 & -0.021 & 0.011 & 0.011 \\
-0.013 & -0.021 & 0.042 & -0.020 & -0.021 \\
0.024 & 0.011 & -0.020 & 12.57 & -12.49 \\
0.006 & 0.011 & -0.021 & -12.49 & 12.51
\end{array}\right) \\
&
\end{aligned}
$$

The corresponding correlation matrix is

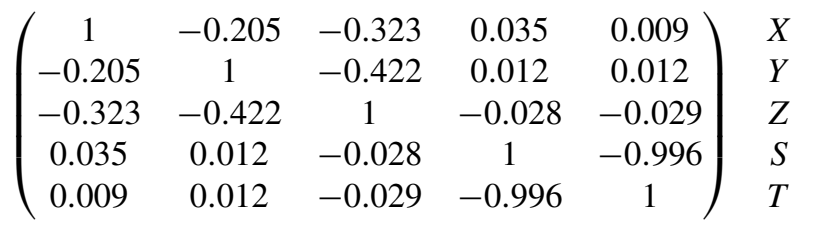

The nearly $-100 \%$ correlation between $S={ }^{e} A$ and one or the other ${ }^{2} \mathrm{D}^{\mathrm{o}}$ energy levels is due to the fact that $S={ }^{e} A$ is essentially determined by subtracting one of the ${ }^{2} \mathrm{D}^{\circ}$ energies from the corresponding $\mathrm{E}$ or $\mathrm{F}$ threshold. As a consequence, any increase (resp. decrease) in the energies of the ${ }^{2} \mathrm{D}^{\mathrm{o}}$ levels will result in an identical lowering (resp. rise) of the ${ }^{4} S_{3 / 2}^{o}$ detachment threshold, as could be guessed from figure 1 .

The optimized set of independent parameters $\boldsymbol{X}$ can be used to readjust the experimental data, according to formula
Table 4. Comparison of every LPT-measured photodetachment threshold of $\mathrm{P}^{-}$[ALA+07] with the corresponding value reconstructed in the present work, and the merged result. All energies are given in $\mathrm{m}^{-1}$ with $2 \sigma$ uncertainties.

\begin{tabular}{llll}
\hline Initial level & $\begin{array}{l}\text { LPT measurement } \\
{[\text { ALA+07] }}\end{array}$ & Present work & Merged results \\
\hline${ }^{3} \mathrm{P}_{0}$ & $575840(40)$ & $575793(8)$ & $575795(8)$ \\
${ }^{3} \mathrm{P}_{1}$ & $584100(40)$ & $584090(8)$ & $584092(8)$ \\
${ }^{3} \mathrm{P}_{2}$ & $602240(100)$ & $602179(8)$ & $602181(8)$ \\
\hline
\end{tabular}

$\boldsymbol{M}_{\mathrm{adj}}=\boldsymbol{I} \boldsymbol{X}=\boldsymbol{I}\left({ }^{t} \boldsymbol{I N I}\right)^{-1}{ }^{t} \boldsymbol{I N} \boldsymbol{M}_{\mathrm{exp}}$. The result of this readjustment is given, for the thresholds measured in this work, by the third column of table 2 . The absolute value of the readjustment in this case never exceeds $0.04 \mathrm{~m}^{-1}$, which shows the excellent internal consistency of our threshold measurements. The covariance matrix associated with $\boldsymbol{M}_{\text {adj }}$ is just $\boldsymbol{I}\left({ }^{t} \boldsymbol{I N I}\right)^{-1 t} \boldsymbol{I}$.

\subsection{Comparison with former spectroscopic data}

4.3.1. Detachment thresholds and the electron affinity ${ }^{e} A(\mathrm{P})$. The detachment thresholds from all three fine-structure levels of $\mathrm{P}^{-}$can be revisited, as shown in table 4 . All three thresholds appear a little lower than what had been measured in the 2007 LPT experiment [ALA+07], but no further than two standard deviations, which keeps the present and former measurements quite compatible. The accuracy of the binding energies of the fine-structure levels of $\mathrm{P}^{-}$is improved by a factor of 5 for the ${ }^{3} \mathrm{P}_{0}$ and ${ }^{3} \mathrm{P}_{1}$ levels, and a factor of 12 for the groundlevel ${ }^{3} \mathrm{P}_{2}$; the electron affinity ${ }^{e} A(\mathrm{P})$, defined by this very binding energy, is $602179(8) \mathrm{m}^{-1}$ or $0.746607(10) \mathrm{eV}$. All uncertainties have been augmented by $0.1 \mathrm{~m}^{-1}$ to account for a possible systematic error on wavenumber measurements, but that does not change the result at this level of uncertainty.

Since our photodetachment experiment does not produce ground-level ${ }^{4} S_{3 / 2}^{o}$ phosphorus, getting back to this minimum detachment energy has required that we include Svendenius's P I spectroscopic data [Sve80] in the analysis. The results of Andersson et al's LPT experiments that we have just compared to our measurements can also be merged in this study, in a more general statistical analysis of the spectroscopic system described by figure 1. Because of the large factor we have gained in accuracy, this only results in a slight shift of ${ }^{e} A(\mathrm{P})$ to $602181(8) \mathrm{m}^{-1}$, with a slight improvement of its precision, when rounded to one $\mu \mathrm{eV}$, namely $0.746609(9) \mathrm{eV}$.

4.3.2. The fine structure of $P^{-}$. The ${ }^{3} \mathrm{P}_{1}-{ }^{3} \mathrm{P}_{2}$ and ${ }^{3} \mathrm{P}_{0}-{ }^{3} \mathrm{P}_{1}$ fine-structure intervals of $\mathrm{P}^{-}$are parameters $X$ and $Y$ of our adjustment procedure, the unrounded values of which can be read in table 3 . Here the output does not depend on another experiment, so these intervals can be given with a sub- $\mathrm{m}^{-1}$ precision. Table 5 shows the result with a comparison to the formerly known values.

4.3.3. The first excited term of $P I$ and its ${ }^{2} D^{\mathrm{o}}$ fine structure. Again our experiment has nothing to say about the mean position of the ${ }^{2} \mathrm{D}^{\mathrm{o}}$ term with respect to the ground level of 
Table 5. The fine-structure of $\mathrm{P}^{-}$, compared to previous measurements. All energies are given in $\mathrm{m}^{-1}$ with $2 \sigma$ uncertainties. For the determination of these intervals, nothing but our measurements need to be taken into account, and the obtained precision makes the result insensitive to merging with previous LPT measurements.

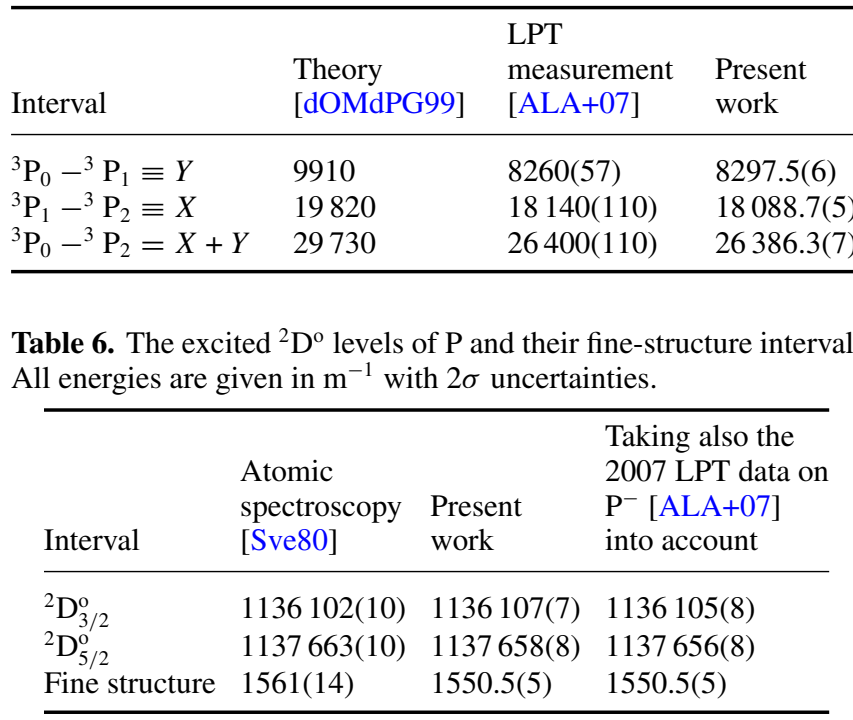

P. Threshold differences however give a good measure of the ${ }^{2} \mathrm{D}^{\mathrm{o}}$ splitting. Since this splitting appears significantly smaller than the difference between the ${ }^{2} \mathrm{D}_{5 / 2}^{\mathrm{o}}$ and ${ }^{2} \mathrm{D}_{3 / 2}^{\mathrm{o}}$ energies found in the literature, the result of the combined data analysis is a symmetric shift of these levels, with the $5 / 2$ and $3 / 2$ levels lowered and raised, respectively, by the same quantity (the exact value can be read from table 3). Andersson et al's LPT data [ALA+07] break this symmetry with a measurement of ${ }^{e} A(\mathrm{P})$ that pushes the ground level of $\mathrm{P}$ a little higher in the $\mathrm{P} / \mathrm{P}^{-}$system depicted in figure 1 . Merging our results with those of the 2007 LPT experiment thus results in an additional downshift of both fine-structure levels by the same quantity, as shown in table 6 .

The amplitude of the corrections to be applied to the ${ }^{2} \mathrm{D}^{\mathrm{o}}$, especially the $J=5 / 2$ one, strongly suggests that the standard deviation of these levels was actually closer to 5 than to $1 \mathrm{~m}^{-1}$. A non-zero covariance of these fine-structure levels could of course change their reaction to the additional constraints introduced here, but the spectroscopic study of the P I spectrum does not reveal anything about it. The measurement of the finestructure splitting of the ${ }^{2} \mathrm{D}^{\mathrm{o}}$ term of $\mathrm{P}$ appears as the most precise result of the present study, which is consistent with the fact that all of our excitation schemes ended on one or the other of the concerned levels, hence contributed to measuring this doublet interval. The improvement brought forward here with respect to the interval extracted from the neutral atom spectroscopy is at least a factor of 20 in accuracy.

\subsection{Hyperfine structures}

Accurately fitting the photodetachment interferograms requires that the synthetic images used for that purpose take all of the imperfections of the image formation into account. Fitting images, such as the one shown in figure 2, are actually

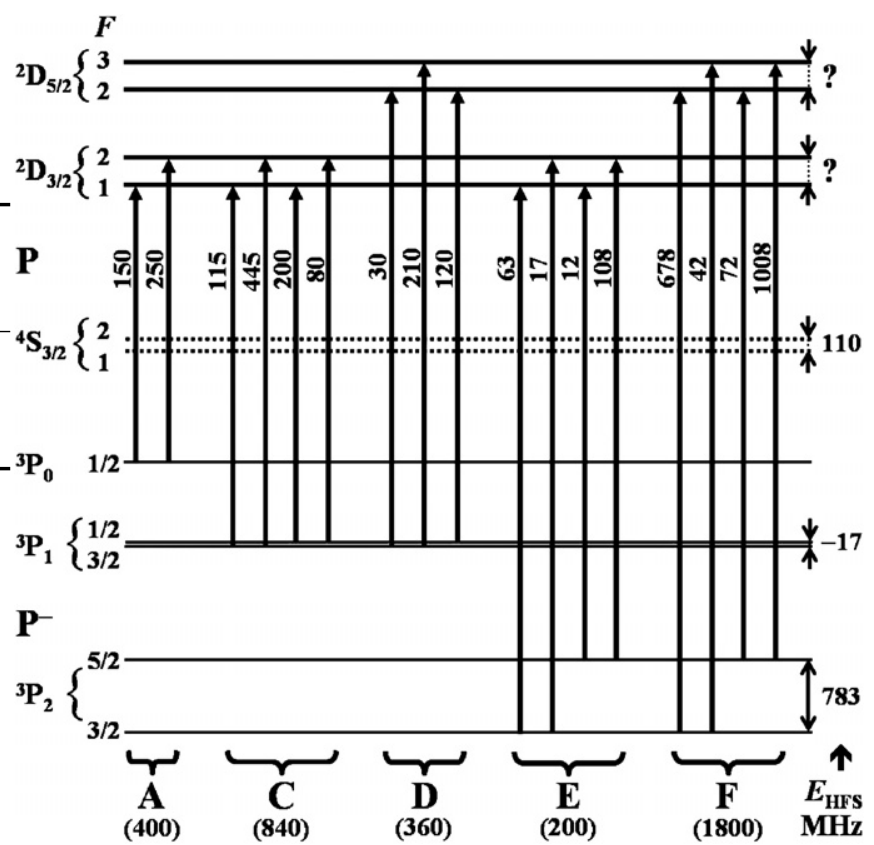

Figure 4. The complete fine- and hyperfine structure of the $\mathrm{P}^{-3} \mathrm{P} \rightarrow \mathrm{P}^{2} \mathrm{D}$ detachment thresholds, with their relative intensities (to be divided by 3600 if one wants a sum equal to 1 ). The hfs intervals indicated for the ${ }^{3} \mathrm{P}_{2}$ and ${ }^{3} \mathrm{P}_{1}$ levels of $\mathrm{P}^{-}$are just extrapolations, from the calculated hfs of isoelectronic ${ }^{33} \mathrm{~S}$ (see text).

Table 7. Observed spectral half-widths of the studied thresholds with standard deviation $(1 \sigma)$, in $\mathrm{GHz}$.

\begin{tabular}{ccccc}
\hline $\mathrm{A}$ & $\mathrm{C}$ & $\mathrm{D}$ & $\mathrm{E}$ & $\mathrm{F}$ \\
\hline $0.9(4)$ & $0.8(3)$ & $1.7(4)$ & $1.1(4)$ & $1.2(4)$ \\
\hline
\end{tabular}

convolutions of an ideal, $100 \%$ contrasted image, with spatial and spectral apparatus functions.

A reward of these actually time-consuming convolutions is that fitting our photodetachment microscopy images, we also obtain a measurement of their spectral width, the principal origin of which is the Doppler broadening produced by the distribution of light wave-vector/ion velocity angles. On our beam, with a $1.2 \mathrm{keV}$ kinetic energy, the Doppler half-width for the detachment photon energy typically amounts to $0.5 \mathrm{GHz}$, as shown by past experiments.

Unresolved hyperfine structures get convoluted with this primary source of spectral broadening. The question we have examined is whether the measured spectral widths of the five studied detachment thresholds of $\mathrm{P}^{-}$can tell us something about the underlying hyperfine splittings. The measured values of these spectral widths are given in table 7 .

What we know about the hyperfine splittings, actually little, is shown in figure 4 and remains conjectural. The relationship between the magnetic dipole factors of different fine-structure levels stemming from the same electron configuration [Gou31] may help to reduce the number of unknowns. But the uncertainties are so large that all of the attempts that we have made to fit these five measured half-widths with a reduced number of free parameters led to inconclusive results. It of course appears natural that the 
images obtained at the $\mathrm{E}$ and $\mathrm{F}$ thresholds have a greater halfwidth because of the particularly large hfs splitting of the ${ }^{3} \mathrm{P}_{2}$ level, but the smallest width obtained at threshold $\mathrm{C}$ on the one hand, the largest width obtained at threshold D on the other hand remain a puzzle, if the hfs of both ${ }^{2} \mathrm{D}_{J_{2}}$ levels are to be of the same order of magnitude. More conclusive results on the hyperfine structure of this $\mathrm{P}^{-3} \mathrm{P} \rightarrow \mathrm{P}^{2} \mathrm{D}$ system are thus left to further studies.

\section{Conclusion}

Photodetachment microscopy to an excited state has produced information on the internal spectrum of $\mathrm{P}^{-}$, the electron affinity of phosphorus and even the spectroscopy of the neutral atom, with a 20 times improvement in accuracy for the first finestructure doublet. The electron affinity ${ }^{e} A(\mathrm{P})$ is now known with a $\pm 8 \mathrm{~m}^{-1}$ accuracy, only limited by the present state of the accuracy of neutral P spectroscopy. Should this accuracy undergo significant improvements, the present data could be used to determine the electron affinity of $\mathrm{P}$ with a sub- $\mathrm{m}^{-1}$ accuracy. At such a resolution, however it would become necessary to make a distinction between the hfs-averaged electron affinity and the more rigorous, lowest hyperfine level to lowest hyperfine level electron affinity. A more accurate estimate of the hyperfine structure of $\mathrm{P}^{-}$would thus become necessary. Photodetachment microscopy to excited states may also be applied to many other atoms and anions, so as to bring forward comparable improvements in the spectroscopy of both the anion and the first excited levels of the atom.

\section{Acknowledgments}

The authors wish to thank Evelyne Cottereau and Christophe Moreau, at the Laboratoire de mesure du carbone 14 (CEA Saclay), for making their expertise repeatedly available to us during the first months of operation of the SNICS II ion source, and Kevin Glize, for some useful numerical simulations that helped adapting the first decelerator to our low-energy beam requirement. Thanks are also due to Pontus Andersson and Dag Hanstorp for complementing their 2007 results with some unpublished data.

\section{Appendix. Fine- and hyperfine-structure branching ratios}

The branching ratios, or relative intensities, of the photodetachment channels of an atomic system are a little more involved than the relative intensities of a bound-bound transition, because of the additional degree of freedom given by the angular momenta of the outgoing electron. The corresponding formulae for the relative intensities have already been published either when the anion/atom system has a fine[BCDD06] or a hyperfine structure [BDV+01]. For the latter case, a simplification of course occurs when the initial ion is a ${ }^{1} \mathrm{~S}_{0}$ state, as with $\mathrm{I}^{-}$. In this case, the relative intensities are just proportional to $2 F_{2}+1$, with $F_{2}$ the total angular momentum of the residual atom [PBDD09].
In order to compare all components of the $\mathrm{P}^{-3} \mathrm{P} \rightarrow$ $\mathrm{P}^{2} \mathrm{D}$ transition array, we must know the relative intensities of all hyperfine channels, including from one fine-structure component to the other. This appendix gives a more general formulae, valid in all cases where both a fine- and a hyperfinestructure spread the photodetachment probability among a set of different angular momentum components.

The general formalism remains the same as in the quoted papers [PBDD09, BDV+01], with the usual notations $L, S$, $J, I$ and $F$ for the orbital, spin, total electron, nuclear and total angular momentum, respectively. Indices ${ }_{1}$ and 2 are to designate the initial anion and final neutral atom respectively. Quantum numbers $l, s$ and $j$ stand for the orbital, spin and total angular momentum of the photodetached electron. A quantum number $F$ can be introduced for the total angular momentum of the neutral atom+detached electron system.

Following the same lines as when dealing with the hyperfine structure of ${ }^{17} \mathrm{O}^{-}$and ${ }^{17} \mathrm{O}$ and using Racah algebra [RBMW59, Arm71, Jud98], one finds that for s-wave photodetachment $(l=0)$, intensities $I\left(J_{2}, F_{2}, J_{1}, F_{1}\right)$ are such that

$$
\begin{aligned}
& I\left(J_{2}, F_{2}, J_{1}, F_{1}\right) \propto\left[J_{2}, F_{2}, J_{1}, F_{1}\right] \sum_{F}[F] \\
& \quad \times\left\{\begin{array}{llllllll}
s & & F & & 1 & & L_{2} & \\
& F_{2} & & F_{1} & & L_{1} & & S_{2} \\
J_{2} & & I & & J_{1} & & S_{1} &
\end{array}\right\}^{2},
\end{aligned}
$$

where $[J]$ stands for the degeneracy factor $2 J+1$. The standard 12-j symbol of the first kind [Jud98] implies eight triangular conditions, on $\left(S_{1}, L_{1}, J_{1}\right),\left(S_{2}, L_{2}, J_{2}\right),\left(J_{1}, I, F_{1}\right)$, $\left(J_{2}, I, F_{2}\right),\left(S_{2}, s, S_{1}\right),\left(F_{2}, s, F\right),\left(F_{1}, 1, F\right)$ and $\left(L_{1}, 1, L_{2}\right)$.

The branching ratios can also be calculated by the fractional parentage method introduced by Engelking and Lineberger [EL79] where the laser interaction 'annihilates' an electron of spin $s=1 / 2$ and angular momentum $l$ ' and promotes it into the continuum $\left(l^{\prime}=1\right.$ for the 'annihilated' 3 p-electron of $\left.\mathrm{P}^{-}\right)$. This calculation was already described too when applied to the hyperfine structure of the ${ }^{17} \mathrm{O}^{-} \rightarrow{ }^{17} \mathrm{O}$ detachment process $[\mathrm{BDV}+01]$. The relative intensities for s-wave photodetachment appear then as

$$
\begin{aligned}
& I\left(J_{2}, F_{2}, J_{1}, F_{1}\right) \propto\left[J_{2}, F_{2}, J_{1}, F_{1}\right] \sum_{j^{\prime}=\ell^{\prime}-s}^{j^{\prime}=\ell^{\prime}+s}\left[j^{\prime}\right] \\
& \quad \times\left\{\begin{array}{ccc}
F_{2} & j^{\prime} & F_{1} \\
J_{1} & I & J_{2}
\end{array}\right\}^{2}\left\{\begin{array}{ccc}
S_{1} & L_{1} & J_{1} \\
s & \ell^{\prime} & j^{\prime} \\
S_{2} & L_{2} & J_{2}
\end{array}\right\}
\end{aligned}
$$

which implies nine triangular conditions, on $\left(S_{1}, L_{1}, J_{1}\right)$, $\left(S_{2}, L_{2}, J_{2}\right),\left(s, l^{\prime}, j^{\prime}\right),\left(S_{2}, s, S_{1}\right),\left(L_{1}, l^{\prime}, L_{2}\right),\left(J_{1}, j^{\prime}, J_{2}\right)$, $\left(J_{1}, I, F_{1}\right), \quad\left(J_{2}, I, F_{2}\right)$ and $\left(F_{2}, j^{\prime}, F_{1}\right)$. Numerical calculations show that both methods give the same results, which was justified in detail as a comment to the equality observed for the fine-structure case [SG08]. The relative weights of the $\mathrm{P}^{-3} \mathrm{P}_{J_{1} F_{1}} \rightarrow \mathrm{P}^{2} \mathrm{D}_{J_{2} F_{2}}$ photodetachment transitions are given by figure 4 . 


\section{References}

[AHH99] Andersen T, Haugen H K and Hotop H 1999 Binding energies in atomic negative ions: III J. Phys. Chem. Ref. Data 281511

[ALA+07] Andersson P, Lindahl A O, Alfredsson C, Rogström L, Diehl C, Pegg D J and Hanstorp D 2007 The electron affinity of phosphorus J. Phys. B: At. Mol. Opt. Phys. 404097

[Arm71] Armstrong L Jr 1971 Theory of Hyperfine Structure of Free Atoms (New York: Wiley Interscience)

[BGH+95] Berzinsh U, Gustafsson M, Hanstorp D, Klinkmüller A, Ljungblad U and Mårtensson-Pendrill A-M 1995 Isotope shift in the electron affinity of chlorine Phys. Rev. A 51 231-8

[BCD+05] Blondel C, Chaibi W, Delsart C, Drag C, Goldfarb F and Kröger S 2005 The electron affinity of $\mathrm{O}, \mathrm{Si}$, and $\mathrm{S}$ revisited with the photodetachment microscope Eur. Phys. J. D 33335

[BCDD06] Blondel C, Chaibi W, Delsart C and Drag C 2006 The fine structure of $\mathrm{S}$ and $\mathrm{S}^{-}$measured with the photodetachment microscope J. Phys. B: At. Mol. Opt. Phys. 391409

[BDD96] Blondel C, Delsart C and Dulieu F 1996 The photodetachment microscope Phys. Rev. Lett. 773755

[BDDV99] Blondel C, Delsart C, Dulieu F and Valli C 1999 Photodetachment microscopy of $\mathrm{O}^{-}$Eur. Phys. J. D 5207

[BDG01] Blondel C, Delsart C and Goldfarb F 2001 Electron spectrometry at the $\mu \mathrm{eV}$ level and the electron affinities of Si and F J. Phys. B: At. Mol. Opt. Phys. $34 \mathrm{~L} 281$

Blondel C, Delsart C and Goldfarb F 2001 Electron spectrometry at the $\mu \mathrm{eV}$ level and the electron affinities of Si and F J. Phys. B: At. Mol. Opt. Phys. 342757 (erratum)

[BDV+01] Blondel C, Delsart C, Valli C, Yiou S, Godefroid M and Van Eck S 2001 Electron affinities of ${ }^{16} \mathrm{O},{ }^{17} \mathrm{O}$, ${ }^{18} \mathrm{O}$, the fine structure of ${ }^{16} \mathrm{O}^{-}$, and the hyperfine structure of ${ }^{17} \mathrm{O}$ Phys. Rev. A $\mathbf{6 4} 052504$

[CDS+10] Carette T, Drag C, Scharf O, Blondel C, Delsart C, Froese Fischer C and Godefroid M 2010 Isotope shift in the sulfur electron affinity: observation and theory Phys. Rev. A 81042522

[CG11] Carette T and Godefroid M R 2011 Ab initio calculations of the ${ }^{33} \mathrm{~S} 3 p^{43} P_{J}$ and ${ }^{33} \mathrm{~S}^{-} /{ }^{37,35} \mathrm{Cl}$ $3 p^{52} P_{J}^{\mathrm{o}}$ hyperfine structures J. Phys. B: At. Mol. Opt. Phys. 44105001 (arXiv:1101.5645v1 [physics.atom-ph])

[dOMdPG99] de Oliveira G, Martin J M L, de Proft F and Geerlings $P 1999$ Electron affinities of the firstand second-row atoms: benchmark $a b$ initio and density-functional calculations Phys. Rev. A $601034-45$

[DMPD68] Dutta N C, Matsubara C, Pu R T and Das T P 1968 Brueckner-Goldstone many-body theory for the hyperfine structure of phosphorus Phys. Rev. Lett. 21 1139-43

[EL79] Engelking P C and Lineberger W C 1979 Laser photoelectron spectrometry of $\mathrm{Fe}^{-}$: the electron affinity of iron and the 'nonstatistical' fine-structure detachment intensities at $488 \mathrm{~nm}$ Phys. Rev. A 19149
[Fab94] Fabrikant I I 1994 J. Phys. B: At. Mol. Opt. Phys. 274545

[Fel76] Feldmann D 1976 Infra red photodetachment threshold measurements: $\mathrm{Li}^{-}$and $\mathrm{P}^{-}$Z. Phys. A 27719

[Gou31] Goudsmit S 1931 Theory of hyperfine structure separations Phys. Rev. 37 663-81

[GxOT98] Guo-xin C, Ong P P and Ting L 1998 DFT approach for electron affinity of negative ions Chem. Phys. Lett. 290211

[GJB98] Gutsev G L, Jena P and Bartlett R J 1998 Electric quadrupole moments and electron affinities of atoms from $\mathrm{H}$ to $\mathrm{Cl}$ : a coupled-cluster calculation Chem. Phys. Lett. 291547

[Jud98] Judd B R 1998 Operator Techniques in Atomic Spectroscopy (Princeton, NJ: Princeton University Press)

[JP75] Juncar P and Pinard J 1975 A new method for frequency calibration and control of a laser Opt. Commun. 14438

[Kra11] Kramida A E 2011 The program LOPT for least-squares optimization of energy levels Comput. Phys. Commun $\mathbf{1 8 2} 419$

[LP62] Lambert R H and Pipkin F M 1962 Hyperfine structure of atomic phosphorus Phys. Rev. 128 198-201

[LP63] Lambert R H and Pipkin F M 1963 Hyperfine structure of atomic phosphorus Phys. Rev. 1292836 (erratum)

[LML91] Lykke K R, Murray K K and Lineberger W C 1991 Threshold photodetachment of $\mathrm{H}^{-}$Phys. Rev. A 43 6104-7

[PM11] Parthiban S and Martin J M L 2011 Assessment of W1 and $\mathrm{W} 2$ theories for the computation of electron affinities, ionization potentials, heats of formation, and proton affinities J. Chem. Phys. 1146014

[PBDD09] Peláez R J, Blondel C, Delsart C and Drag C 2009 Pulsed photodetachment microscopy and the electron affinity of iodine J. Phys. B: At. Mol. Opt. Phys. 42125001

[PS64] Pendlebury J M and Smith K F 1964 Hyperfine structure measurements in ${ }^{75} \mathrm{As},{ }^{31} \mathrm{P}$ and ${ }^{53} \mathrm{Cr}$ Proc. Phys. Soc. 84 849-56

[RFSG72] Radziemski L J Jr, Fisher K J, Steinhaus D W and Goldman A S 1972 Calculation of atomic energy level values Comput. Phys. Commun. 39

[RBMW59] Rotenberg M, Bivins R, Metropolis N and Wooten J K Jr 1959 The 3-j and 6-j Symbols (London: Crosby Lockwood \& Son Ltd)

[SG08] Scharf O and Godefroid M R 2008 On the fine structure photodetachment intensities using the irreducible tensorial expression of second quantization operators, http://arxiv.org/abs/0808.3529v1

[SL77] Slater J and Lineberger W C 1977 High-resolution photodetachment studies of $\mathrm{P}^{-}$and $\mathrm{Te}^{-}$Phys. Rev. A 15 2277-82

[SV80] Svendenius N and Vergès J 1980 The P I spectrum in the region 1-4 $\mu \mathrm{m}$ Phys. Scr. 22288

[Sve80] Svendenius N 1980 The spectrum and term analysis of neutral phosphorus, P I Phys. Scr. 22240

[TJL89] Trainham R, Jopson R M and Larson D J 1989 Measurement of the hyperfine structure of ${ }^{33} \mathrm{~S}^{-}$ Phys. Rev. A 393223

[WP98] Wijesundera W P and Parpia F A 1998 Negative ions of carbon, nitrogen, and phosphorus Phys. Rev. A 57 3462-8 